\title{
E-Healthcare Billing and Record Management Information System using Android with Cloud
}

\author{
Vanitha $\mathrm{T} \mathrm{N}^{1}$, Narasimha Murthy $\mathrm{M} \mathrm{S}^{2}$, Chaitra $\mathrm{B}^{3}$ \\ ${ }^{I}$ (Fourth Semester M.Tech, Department of Computer Science \& Engineering, Acharya Institute of Technology, \\ Bangalore, India) \\ ${ }^{2}$ (Assistant Professor, Department of Computer Science \& Engineering, Acharya Institute of Technology, \\ Bangalore, India) \\ ${ }^{3}$ (Assistant Professor, Department of Computer Science \& Engineering, Acharya Institute of Technology, \\ Bangalore, India)
}

\begin{abstract}
In today's civilized society, the people are betrayed with proper healthcare facilities. In order to minimize the cost and complexity involved in processing traditional billing system, Electronic health records (EHR) and electronic billing systems have been proposed as mechanisms to help curb the rising costs of healthcare and also helps to detect the fraudulent practices in healthcare system. The introduction of Cloud computing concept in electronic healthcare systems is the solution for better utilization of healthcare facilities. It uses open-source cloud computing technologies as the mechanism to build an affordable, secure, and scalable platform that supports billing as well as EHR operations. We call this platform as "MedBook" which is a cloud solution that provides patients, healthcare providers, and healthcare payers a platform for exchange of information about EHR, billing activities, and benefits inquiries. MedBook serves as an integration point between the various participants in the healthcare delivery system. This paper presents the architecture and implementation status of this system. The developed system has been evaluated using the Jelastic cloud service. MedBook is a Software-as-a-Service (SaaS) application built on top of open source cloud technologies and running on an Infrastructure-as-a-Service (IaaS) platform. The client applications are mobile apps run from Google's Android enabled devices.
\end{abstract}

Keywords - Cloud Computing, EHR, Mobile apps, Open source Cloud, REST-based API, SaaS

\section{INTRODUCTION}

Electronic health records (EHR) and electronic billing systems have been proposed as mechanisms to curb the rising costs of healthcare and also helps to detect the fraudulent practices in the traditional healthcare system[1]. Many healthcare professionals, hospitals and insurance agencies maintains the paper-based records, billing of the patients which is been converted later into computer-based billing and records which can be abused, modified or lost for malpractice done by frauds either for money or grudge.

Hence the personal information of the patients is revealed, bogus information are entered and misused in traditional Healthcare system. Moreover Traditional healthcare system depends on the centralized server which is unreliable, insecure in accessing, storing medical data regardless of time, cost and location. Hence it is more complex and lack privacy and cost involved in integrating medical information is expensive.

Given this scenario, Electronic Health Records (EHR) and Electronic Medical Billing (EMB) have been proposed as a mechanism which reduces healthcare disparities and ensures adequate privacy and security. One potential solution for addressing all aforementioned issues is the introduction of Cloud Computing concept in electronic healthcare systems. This mechanism pursued the idea of using open-source public cloud computing Technologies and mobile plus cloud paradigm [2] to build an affordable, secure and scalable platform that supports billing as well as EHR operations. We call this platform as MedBook and in this paper we present the proposed architecture and implementation status of this system. MedBook is a cloud solution that provides patients, healthcare professionals/providers and healthcare payers a platform for exchange of electronic information about billing activities, benefit inquiries and EHR operations such as insert delete and update record using open source cloud services and Android operating system(OS).

MedBook is Software-as-a-Service (SaaS) platform built on top of open source public cloud technologies and running on the top of an Infrastructure-as-a Service (IaaS) platform [3]. Generally the server applications are implemented as a collection of web services and web applications using MySQL, Tomcat 6or7 server, Apache web server. All the web services run on virtual machines powered by Windows XP or Ubuntu 
Linux 10.04.These servers are hosted inside an open source cloud [4] which can be Jelastic, Eucalyptus 2.0, Open Stack and so forth.

The client applications are mobile apps run from Google's Android Enabled phones [5]. These client applications are built using Java 1.7 or 1.6 and uses REST based API to interact with MedBook SaaS Infrastructure.

The rest of the paper is organized as follows. Section II provides the basics of cloud computing and EHR details. Section III provides the Motivation for the use of MedBook. Section IV presents the proposed system architecture of MedBook SaaS application and its implementation status. Section V discusses the scope of the work. Finally, section VI presents a summary and conclusion of the paper.

\section{Cloud COMPUTING AND ELECTRONiC HEALTH INFORMATION}

According to NIST " Cloud computing is a pay per use model for enabling convenient, on demand network access to a shared pool of configurable computing resources (e.g., networks, servers, storage, applications, and services) that can be rapidly provisioned and released with minimal management effort or service provider interaction[6].

Cloud Computing provides the following Key Characteristics [7]:

(a)Broad network access Capabilities are available over the network and accessed through standard mechanisms that promote use by heterogeneous thin or thick client platforms (e.g., mobile phones, laptops, and PDAs).

(b)Resource pooling The provider's computing resources is pooled to serve multiple consumers using a multitenant model; Multi-tenancy [8] enables sharing of resources and costs across a large pool of users thus allowing for:

- Centralization of infrastructure in locations with lower costs (such as real estate, electricity, etc.)

- Peak-load capacity increases (users need not engineer for highest possible load-levels)

- Utilisation and efficiency improvements for systems that are often only $10-20 \%$ utilised.

Examples of resources include storage, processing, memory, network bandwidth, and virtual machines.

(c)Application programming interface (API) accessibility to software that enables machines to interact with cloud software in the same way the user interface facilitates interaction between humans and computers. Cloud computing systems typically use REST-based APIs [9].

(d) Cost is claimed to be reduced when using cloud infrastructure.

(e)Reliability is improved if multiple redundant sites are used, which makes well-designed cloud computing suitable for business continuity and disaster recovery.

(f)Security could improve due to centralization of data, increased security-focused resources, etc., but concerns can persist about loss of control over certain sensitive data, and the lack of security for stored kernel.

Cloud computing has three service models to deliver various services namely, Software as a Service (SaaS), Platform as a Service (PaaS), Infrastructure as a Service (IaaS) [7].

Software as a Service (SaaS) In this Service model, an application software is hosted in the cloud and thus the users can access the application through Mobile apps, emails, PC's. The users need not have to control the cloud infrastructure since the model itself employs the multi-tenancy system architecture for accessing the application and provides optimization in terms of speed, security, availability, disaster recovery and maintenance. Example of SaaS includes Google Mail, Google Docs, Salesforce.com, ZOHO and so forth.

Platform as a Service (PaaS) In this Service model, the users has to deploy onto the cloud infrastructure created using programming languages and tools and configuration management supported by the provider. The users need not have to control the cloud infrastructure including network, servers, operating systems, or storage. PaaS model offers a development platform to host both the completed and in- progress cloud application. Example of PaaS includes Google AppEngine, AWS.

Infrastructure as a Service (IaaS) In this Service model, the users directly use processing, storage, networks, and other computing resources provided in the IaaS cloud which employs Virtualization in order to integrate/decompose physical resources as per user requirements. The users need not have to manage or control the cloud infrastructure but has control over operating systems, storage, deployed applications. Example of IaaS includes Amazon's EC2, Sun Microsystems.

Cloud computing provides various deployment models they are given as follows, Private cloud, Public cloud, Hybrid cloud and community cloud [7].

Private cloud The cloud infrastructure is operated within a single organization, and it is managed by the organization itself or by a third-party which may exist on/ off premises. 
Public cloud The cloud infrastructure is made available to the general public or a large industry group and is owned by service providers. These services are free or offered on a pay-per-use model. Generally, public cloud service providers like Amazon EC2, S3, Microsoft and Google AppEngine.

Community cloud The cloud infrastructure is shared by several organizations and supports a specific community that has shared concerns (e.g., mission, security requirements, policy, and compliance considerations). It may be managed by the organizations or a third party and may exist on/off premises.

Hybrid cloud The cloud Infrastructure is a composition of two or more clouds (private, community or public) that remain unique entities but are bound together, offering the benefits of multiple deployment models.,

Given the characteristics of Cloud Computing and its service models, thus improves the ability of accessing the information by the users being able to rapidly and inexpensively re-provision technological infrastructure resources. Device and location independence enable users to access systems using a web browser regardless of their location or what device they are using (e.g., mobile phones). Multi-tenancy enables sharing of resources and costs across a large pool of users thus allowing for centralization of infrastructure in locations with lower costs. Reliability improves through the use of multiple redundant sites, which makes Cloud Computing suitable for business continuity and disaster recovery. Security typically improves due to centralization of data and increased security-focused resources. Sustainability comes about through improved resource utilization, more efficient systems.

Electronic health information is accessed by all the participants of healthcare system such as patients, healthcare providers, healthcare payer using open source cloud which acts as a server that faces several challenges, like data storage and management (e.g., physical storage issues, availability and maintenance), interoperability and availability of heterogeneous resources, security and privacy (e.g., permission control, data anonymity, etc.), unified and ubiquitous access.

The mobile apps such as Google's Android operating system is used as a client which focus towards achieving two specific goals [10]: the availability of e-health applications and medical information anywhere and anytime and the invisibility of computing. Mobile apps basically support electronic billing and EHR activities of patient and their medical history which can be accessed individually by patient, healthcare provider, healthcare payer by authenticating themselves with MedBook cloud server.

\section{Motivation}

MedBook provides a highly reliable and secure electronic billing and record management system. It also helps reduce the occurrences of medical errors due to incomplete medical information. Privacy of medical information is maintained to prevent unauthorized access and misuse of electronic information [11].Since the MedBook is Mobile plus cloud paradigm, the various participants such as patients, health care payers, healthcare professional can exchange information regardless of time, location, cost involved in it. Since the proposed system utilizes open source cloud computing technologies, the interaction of healthcare participants with MedBook SaaS application can be done globally which reduce the cost associated in accessing medical information to certain limit [12]. In addition, by integrating and correlating the billing system with EHR, it becomes possible to find that a given procedure was actually performed or the medical history of the patient utilized such procedures.

\section{Proposed System Architecture and Implementation Details}

Fig. 1 Depicts the MedBook proposed system architecture. MedBook SaaS application serves as an integration point between the various participants in the healthcare delivery system such as Patients, healthcare providers, healthcare payer [3]. MedBook architecture basically contains two Modules such as Client Module which uses the Mobile apps such as android enabled phones to interact with MedBook application. Server Module which consists of a series of web services and databases residing inside an IaaS cloud that maintains the information about each patient's EHR. 


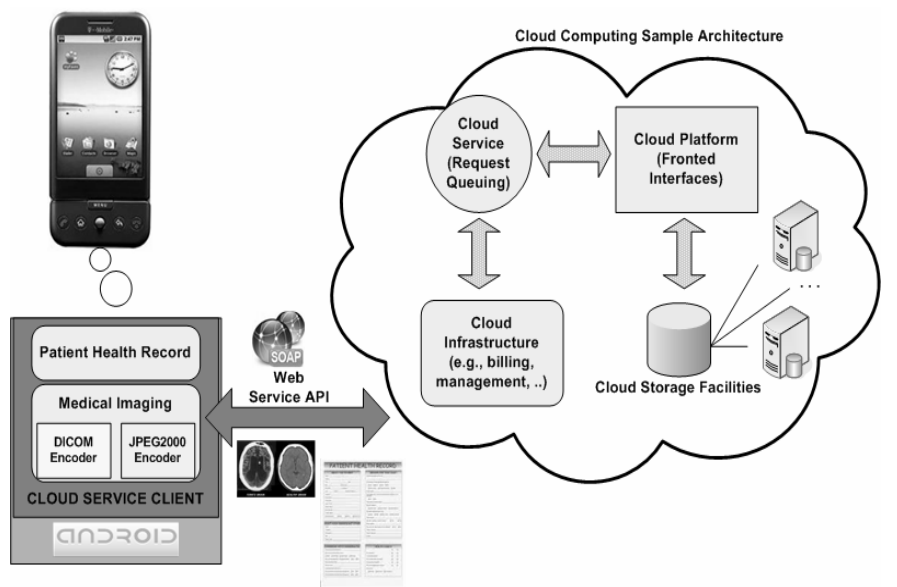

Fig. 1 MedBook proposed system architecture

\section{A. MedBook Client Application}

The MedBook Client applications are mobile apps which uses Android enabled phones that connect with the MedBook SaaS infrastructure by means of REST based API. The architecture of MedBook Client application is depicted in Fig. 2. An android enabled phone is an open source and basically supports large number of applications compared to other Smart phones [13]. Android client application is designed for Patients, Healthcare provider/professional, Healthcare payers to perform billing and EHR activities with MedBook.

The Android client application for patient is designed to perform following operations to:

- Access Medical details prescribed

- Know Insurance Benefit plan

- Access their EHR Information

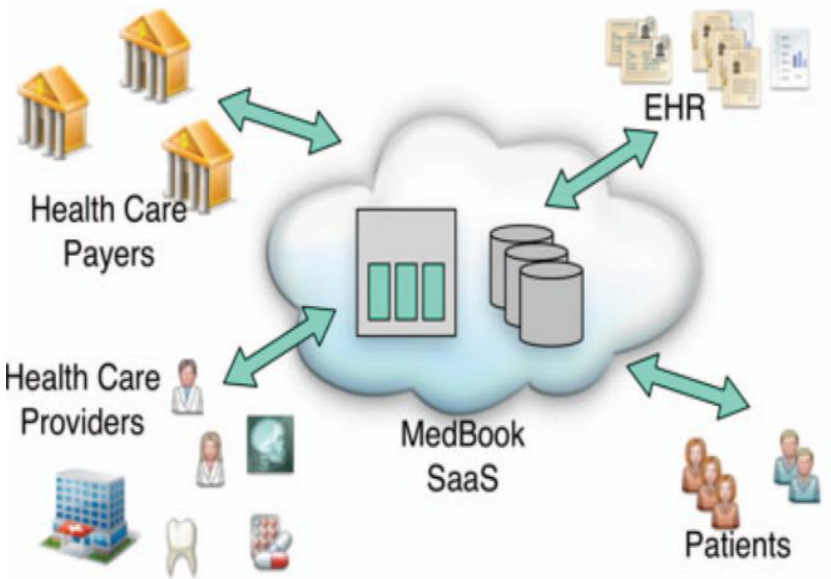

Fig. 2 MedBook Client Architecture

The Android client application for Healthcare payers is designed to perform following operations:

- To receive billing request

- Response to Benefit and Enrollment verification request

- Response to authorization request for procedures/drugs

- To enter Insurance Benefit plan details of a particular patient

The Android client application for Healthcare provider/professional is designed to perform following operations to:

- Submit billing Claims

- Get status information on claims

- Manage EHR of the patient i.e., access and update 
- Request authorization for treatment/drugs

- Enter patient medical details

- Submit benefit enquiries

\section{B. MedBook Server Application}

MedBook consists of a series of web services and databases residing inside an IaaS cloud that maintains the information related to each patient's EHR activities [14]. The architecture of MedBook server application is depicted in Fig. 3. The server module is designed to perform following activities such as

- Service description

- Submitting Billing Transactions

- Representing and accessing EHR's

Service description The description of the services used in the MedBook SaaS application is as follows [3]:

(a)Billing Collector This web service receives the list of billing requests from the healthcare professionals on behalf of patient, and sends to the billing service instances for validation and further processing done by healthcare payers.

(b)Billing Service This web service is an interface between the healthcare payers with respect to healthcare provider and patient. It performs the verification operation on the billing request and reports back to healthcare provider about errors or omissions. Successfully validated claims are sent to the healthcare payer. This service also receives notifications from the healthcare payer (through payer gateway service) regarding the queries related to Patient medical history. It also provides notifications to the healthcare providers or patient which can be fetched by the client application.

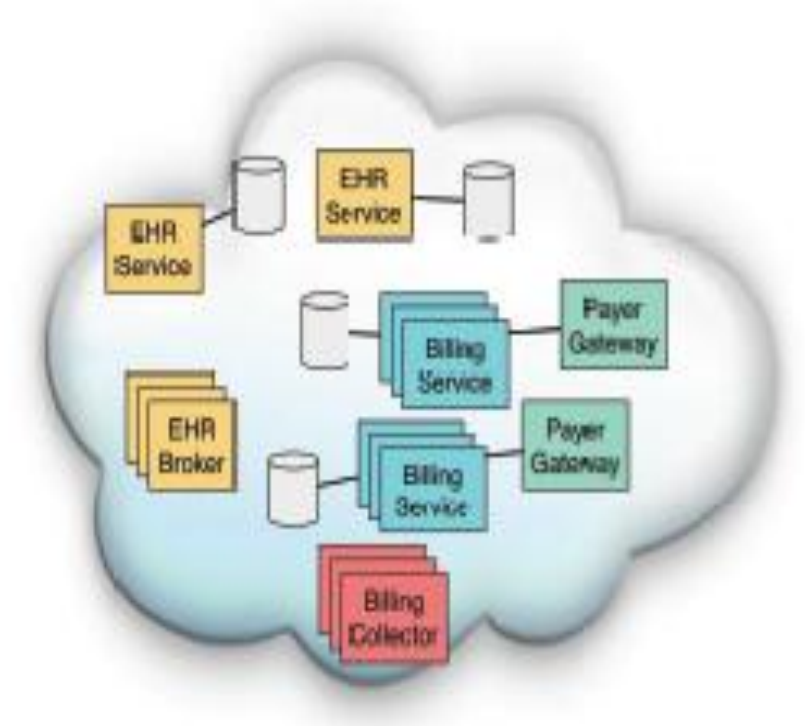

Fig. 3 MedBook Server Architecture

(c)EHR broker This web service takes care of locating all the patient medical history through EHR. Since each EHR is given a unique Patient identification number (PIN) to identify the EHR of the patient and a copy of the PIN. This EHR broker can be contacted by the client application or by the other web services such as the billing service or the payer gateway.

(d)EHR Service This web service provides read-write-update operations for a collection of the EHR segments. EHR service communicates securely by means of encrypted HTTP channels.

(e)Payer Gateway This web service provides an Interface to the healthcare payer to communicate with other services. This service translates all the notifications and request made by the MedBook to native formats used by the healthcare payer. Hence the payer gateway provides a common set of operations and vocabulary to be used between MedBook and the healthcare payers to perform this translation.

Submitting Billing Transactions Typically electronic transactions for medical domains can be classified for the following purpose such as

(a)Enrollment and Benefits Verification used to verify the patient's EHR in a health plan and check whether the patient is valid/Invalid for accessing the benefits of healthcare plan by the healthcare payer from patient's EHR. 
(b)Healthcare Claim used to submit the actual bill of each patient from healthcare provider to healthcare payer for the prescriptions or procedures used in the treatment of patient.

(c)Healthcare Claim status and Notification used to verify the authorization request for procedures is submitted successfully or not to healthcare payer and also receives notification regarding healthcare claim that the billing request is valid or invalid by healthcare payer to the healthcare provider.

Representing and accessing EHR's In MedBook system, each patient has a unique identification number called the Patient Identification Number (PIN) [14] that is used to uniquely identify their EHR. MedBook admin has only the rights to delete a particular EHR when not necessary.EHR is considered as a validation tool against billing transactions submitted for medical procedures. Billing systems should be used with EHR systems because the procedure used by the patient becomes the part of the claims and these data should reflect in patient's EHR also.

Thus the use of EHR has strived for simplicity, scalability and security purpose. In our MedBook system, the portion of the EHR is called Segment and they are stored in a collection of cloud-resident relational database instances. The healthcare provider in MedBook system has control over the segment to render services to patient and the patient manages the demographic information as well as personal information through unique ID and password. Scheme is depicted in Fig. 4. Whenever the healthcare provider adds a new segment into the EHR of a patient two actions are performed firstly the entry with the information about conditions, diagnosis and treatments are stored in the database [15]. This is done through the EHR service and thus EHR is effectively updated to the local segment of the database. Secondly EHR service sends a message to the EHR broker to add a new entry to the segment indexing service which includes the form: (PIN, segment id, database id). Here the database id is a unique id for the database instance that stores the new segment created by the healthcare provider. The EHR broker then delivers all these entries to the client application for display or analysis purpose.

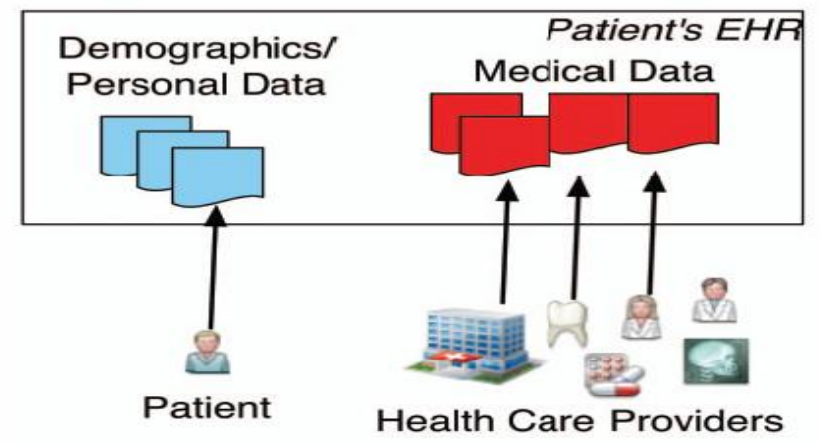

Fig. 4 EHR Control Split between healthcare participants

In our MedBook system, billing process starts when the Billing Collector receives one or more billing transactions. The Collector accepts claims from healthcare providers either as individual transactions or in a batch/queue system. Billing service instance takes the billing job out from the queue system and begins working on each claim. The first task of the billing Service is to verify that the claim is valid or invalid and stores them in a database which the healthcare provider can fetch and correct them for resubmission. Successfully validated claims are also stored into a database and submitted to a Payer Gateway which is then translated into the format used by the internal billing systems to be used by the healthcare payer.

\section{Implementation status}

All the web services were implemented following the Model-View-Controller (MVC) pattern. We used XML to write and deploy the services. All storage is provided using MySQL. All the web services are run on virtual machines powered by Windows XP and all these machines are hosted within an open source cloud namely Jelastic cloud[16][17]. The client applications are Mobile apps such as Android 3.0 and above enabled phones.

\section{Utilizing Jelastic Cloud Service}

For the realization of the mobile healthcare information management system, the Jelastic open source cloud service has been utilized [17]. The main reason for selecting the specific Cloud Computing platform is that it is a commercial service well established and used successfully in several applications. It provides users with several interoperable web interfaces for managing data (SaaS model) and developers with the ability to create their own applications for accessing the latter (IaaS model) and is suitable for managing healthcare information. It provides the technologies necessary to build both private and public clouds, with a goal of standardizing cloud computing infrastructure. 


\section{Scope of Work}

The proposed work helps to curb the rising cost of healthcare to certain limit using open source cloud technologies [18]. Our MedBook system provides solutions to certain top issues in healthcare sector. Firstly, Lack of Information exchange between medical practitioners. Secondly, privacy of Information to prevent unauthorized disclosure of information. Thirdly, Security of IT systems to prevent healthcare data being stolen or intentionally corrupted. Fourthly, accuracy of data to prevent incorrect information to be entered into the EHR.

\section{Conclusion}

Electronic health records (EHR) and Electronic Billing Systems have been proposed as mechanisms to help curb the rising costs of health care system. Given this scenario, the paper discusses the idea of using opensource cloud computing technologies as a cloud solution to build an affordable, secure, and scalable platform that supports billing as well as EHR operations which utilizes Jelastic open source cloud storage service. We call this platform as MedBook, and in this paper we presented the architecture and implementation status of this system. MedBook serves as an integration point between the various participants such as patients, health care providers, and health care payers a platform for exchange of information about EHR, billing activities, and benefits inquiries in the healthcare delivery system.

The proposed system namely, MedBook SaaS application uses open source public cloud services, hence the participants of the healthcare system can access information and relevant healthcare facilities regardless of time, location, cost and privacy factors involved in it. The sharing of medical information resources (electronic health data and corresponding processing applications) is a key factor playing an important role towards the successful adoption of Cloud Computing with Android enabled phones in healthcare systems.

\section{References}

[1] A.S. Boranbayev, and S.N. Boranbayev, "Development and Optimization of Information Systems for Health Insurance Billing", 2010 IEEE $7^{\text {th }}$ International Conference on Information Technology: New Generation, pp - 606 - 613, dated: 12-14 April 2010, Las Vegas, NV.

[2] AM.Rodriguez et.al.’Open911: Experiences with the Mobile Plus Cloud Paradigm”, in Proc.2011 IEEE Cloud Computing Conference, dated: 4-9 July 2011, Washington, DC,USA.

[3] Manuel Rodriguez-Martinez, Harold Valdivia, Jose Rivera, Jaime Seguel, Melvin Greer, "MedBook: A Cloud-based Healthcare Billing and Record Management System”, 2012 IEEE Fifth International Conference on Cloud Computing, pp- 899 - 905, dated: 2429 June 2012 , DOI 10.1109/CLOUD.2012.133

[4] Joe Brockmeier, community evangelist for Cloud Stack Citrix" What is an Open source cloud? ' released on March 28,2013.URL: http://www.linux.com/news/featured-blogs/196-zonker/711498-what-is-open-source-cloud-

[5] "Android Overview" Open Handset Alliance, retrieved on: 15-02-2012.URL:http://www.openhandsetalliance.com/

[6] P. Mell and T. Grance, "Draft NIST working definition of cloud computing - v15," National Institutes of Standard and Technology, Information Technology Laboratory, dated: 19th, August-2009.

[7] Tharam Dillon, Chen Wu and Elizabeth hang,"Cloud computing; Issues and Challenges", AINA, pp.27-33, dated: 20-23, April- 2010, $24^{\text {th }}$ IEEE International Conference, Perth, WA.

[8] A white paper from Juniper Networks Inc on "Securing Multitenancy and cloud Computing", Juniper Networks Inc 2012.pp.1-5,URL: http://www.juniper.net/us/en/local/pdf/whitepapers/2000381-en.pdf

[9] Ben Ramsey "Designing RESTful Web applications" released on September 13 , 2007. http://files.benramsey.com/talks/2007/phpworks/phpworks07-rest.pdf

[10] Alvin Ybanez "Best Android apps for personalizing and customizing your phone" release on 2012-07-13. Retrieved on 09-11-2012. URL: http://www.androidauthority.com/best-apps-customizing-personalizing-android-phones-100685

[11] L. S. Liu, P. C. Shih, G. R. Hayes, "Barriers to the adoption and use of personal health record systems", in Proc. 2011 ACM conference, New York, NY.

[12] S. M. Thompson, and M. D. Dean, "Advancing information Technology in health care", Communications of the ACM, vol. 52, no. 6, 2009.

[13] Palo Alto, Singapore and Reading (UK)"Google's Android becomes the world's leading smart phone platform" Canalys Research release on. January 31, 2011. Retrieved on 15-02-2012.URL:http://www.canalys.com/newsroom/googles-android-becomes-worldsleading-smart-phone-platform

[14] N. Stafford, "Who owns the data in an Electronic Health Record?" EHR Institute, Retrieved on February 29, 2012.URL: http://www.ehrinstitute.org/articles.lib/items/who-owns-the-data-in

[15] R. Green, "Obstacles to EHR Adoption Lie in Small Group Practices". https://www.gplus.com/telecommunicationsservices/Insight/obstacles-to-ehr-adoption-lie-in-small-group practices- 38092, retrieved on: February 28, 2012.

[16] Why Jelastic? Because it's easy! http://jelastic.com/why

[17] "Jelastic cloud hosting", URL: http://www.layershift.com/hosting-services/jelastic-cloud-hosting

[18] Sebastian Rupley "Top Open source resources for cloud computing” released on Nov 6, $2009 . \quad$ URL: http://gigaom.com/2009/11/06/10-top-open-source-resources-for-cloud-computing 